\section{Single-Molecule DNA Stretching Using Optical Tweezers}

Joost van Mameren, Anna Wozniak, and Sid Ragona* JPK Instruments, Berlin, Germany, ${ }^{*}$ Ragona Scientific, Pittsford, NY sid2006@rochester.rr.com

\section{Motivation}

The advent of techniques to mechanically manipulate single (bio)molecules has sparked large efforts to precisely study the mechanical and elastic properties of proteins, protein fibers, DNA, RNA, etc. Two widely used techniques in this area are atomic force microscopy (AFM) and optical tweezers. Optical tweezers complement AFM at the lower end of the force regime: forces of typically a few hundred picoNewtons down to fractions of a picoNewton can be assessed using optical tweezers. This has allowed for, among other things, the precise measurement of forces and displacements exerted by individual motor proteins. In this report, we focus on the use of optical tweezers for force spectroscopy on single DNA molecules, and on the range of applications that this technique offers to learn not only about DNA itself, but also about the mechanics and thermodynamics of protein-DNA interaction.

DNA is a so-called semi-flexible polymer. This means that it has an intrinsic stiffness and therefore resists sharp bending. From the physics point of view, the elasticity of DNA therefore influences its dynamics in an interesting manner. Biologically speaking, the elasticity of DNA affects a wide variety of cellular processes, including protein-induced DNA bending, twisting, or looping.

Since the first single-molecule stretching experiments were performed [1], many reports on the elasticity of double-stranded DNA (dsDNA) have appeared, including many model studies that now encompass most of the experimental data. The unique helical structure formed by two intertwined and base-paired strands (see Figure 1) determines its elastic behavior, including the intriguing phase transition called 'overstretching. During this transition, DNA gains almost a factor two in length without the need to pull harder. Below, we will demonstrate these individual regimes experimentally.

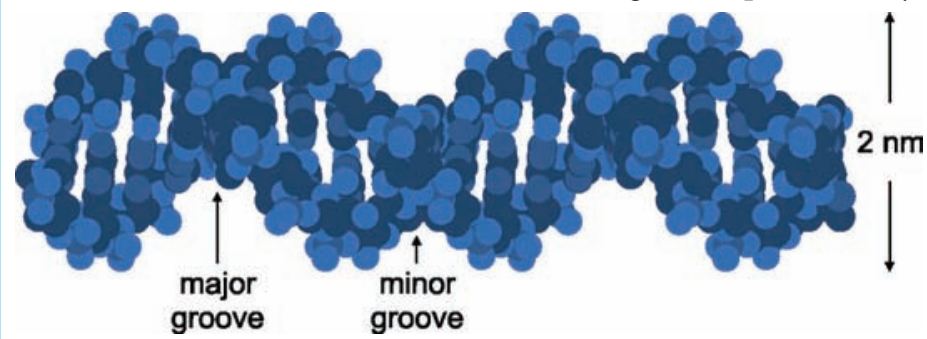

Figure 1: DNA structure. Double-stranded DNA. Two single strands, held together by hydrogen bonds between individual bases, are wound around each other, giving rise to major and minor grooves.

\section{Experiment description}

We have performed our measurements of DNA elasticity using JPK's NanoTracker ${ }^{\text {rit }}$, an optical-tweezers platform designed for quantitative nanomanipulation (Figure 2). The NanoTracker ${ }^{\mathrm{rm}}$ is equipped with two optical traps, which can be both independently steered and used for tracking or force measurements. The two optical traps can be used to suspend a single DNA molecule between two functionalized polystyrene beads, using a multichannel flow cell (see Figure 3a).

By controllably changing the distance between the two traps at the DNA's extremities, we can apply tension to the DNA. Since

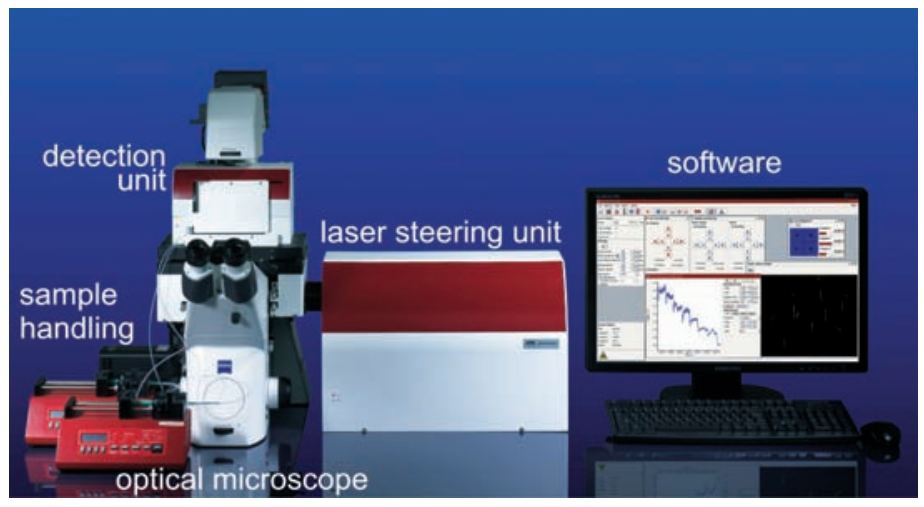

Figure 2: JPK NanoTracker ${ }^{\mathrm{Tn}}$, a dual-beam force-sensing optical tweezers platform in the configuration used for the measurements described here.

the NanoTracker ${ }^{\text {rut }}$ was designed as a force-sensing optical-tweezers instrument, one can accurately measure the forces applied. We can thus measure the 'force versus extension' characteristic of a single DNA molecule, i.e., its elastic response to forces.

Figure 4 shows a typical measurement of the highly non-linear elasticity of double-stranded DNA (samples generously provided by Dr. A. Sischka). Even when the DNA is slack, i.e., when the DNA ends are much closer together than the length of the DNA polymer, a finite force has to be exerted in order to fix its ends. This finite force is of entropic nature: due to the continuous bombardment by solvent molecules, the DNA actually 'wants to' attain as many conformations as possible. Keeping the DNA at a fixed end-to-end distance, one precludes a tremendous number of conformations, lowering the entropy of the DNA. The further one pulls, the more conformational states are precluded, and hence the higher this entropic force is. When the DNA is (almost) pulled taut, one actually starts to increase the length of the DNA contour by stretching the DNA backbone. This stretching occurs linearly with extension, as for a 'Hookean' spring. This regime is called the enthalpic regime. This regime tells us the intrinsic spring stiffness of a DNA molecule.

A remarkable feature is observed at a force of $\sim 65 \mathrm{pN}$ : without the requirement of additional force, the DNA lengthens by almost a factor two. This characteristic phase transition, first reported during the late nineties by two groups in the same issue of Science [2,3], is called the overstretching transition. This transition reproducibly occurs at the same pulling force and is reversible. When the DNA is extended beyond $\sim 170 \%$ of its contour length, the transition is complete and a steep increase in force is observed. Much debate exists in the literature about the structural nature of the transition.
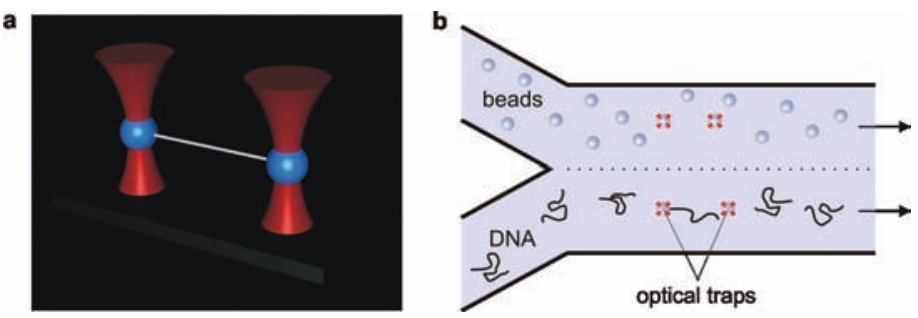

Figure 3: Experimental approach. (a) Schematic illustration of a DNA molecule captured and stretched between two traps. (b) The actual construction of such a 'dumbbell' is facilitated by the use of a multichannel flow cell. Typically, one first captures two handle beads and then swiftly brings these into a flow cell channel with DNA molecules, fed by the syringe pumps of Figure 2. The molecules are specifically labeled on their ends to make them stick to the beads. A connection between trapped beads is then made by moving one of the traps, essentially 'fishing' for the other end. 

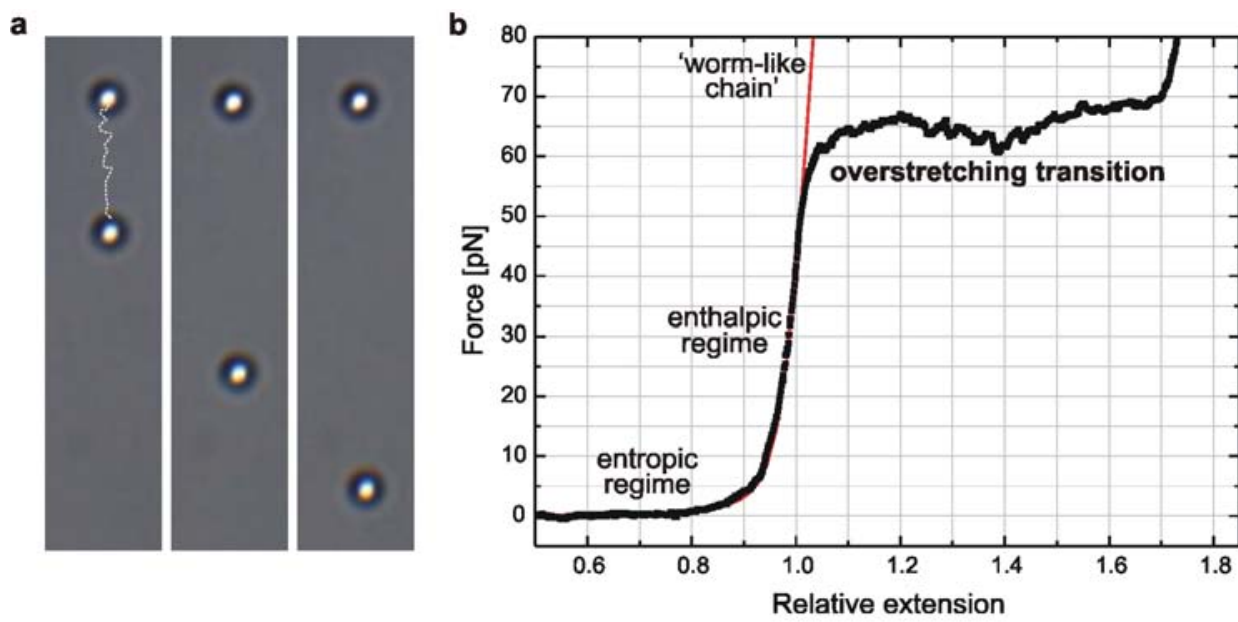

molecules. Science, 271(5250):795-799, 1996.

[3] P. Cluzel, A. Lebrun, C. Heller, R. Lavery, J. L. Viovy, D. Chatenay, and F. Caron. DNA: An extensible molecule. Science, 271(5250):792-794, 1996.

[4] I. Tinoco Jr., P. T. X. Li, and C. Bustamante. Determination of thermodynamics and kinetics of RNA reactions by force. Quarterly Reviews of Biophysics 39(4):325-360, 2006

[5] P.Bechtluft, R.G.H. van Leeuwen, M. Tyreman, D. Tomkiewicz, N. Nouwen, H.L. Tepper, A.J.M. Driessen, S.J. Tans. Direct observation of chaperone-induced changes in a protein folding pathway. Science 318: 1458-1461 (2007).

[6] K. Pant, R. L. Karpel, and M. C. Williams. Kinetic Regulation of Single DNA Molecule Denaturation by T4 Gene 32 Protein Struc-

Figure 4: DNA elasticity measurement using JPK's NanoTracker ${ }^{\mathrm{tw}}$. (a) Microscopy images of the pulling experiment (the DNA is not visible). (b) Force-extension graph of the experiment in panel $a$. As the DNA is pulled to larger extensions, it undergoes several phases of distinct elastic behavior. The red line is a fit to the so-called worm-like chain model [1,2]. The overstretching transition, typical for double-stranded DNA, is readily seen. The DNA samples used were generously provided by Dr. A. Sischka (Anselmetti lab).

More and more evidence exists, however, for the transition being force-induced melting, i.e., gradual breakdown of the interaction between the two strands.

\section{What can we learn? Applications}

Single-molecule force spectroscopy can teach us a lot about the intrinsic mechanical properties of (bio)molecules. The intricate elastic response of DNA, as described here, has been elucidated largely using optical tweezers based technology. Moreover, protein and RNA structure and dynamics can be unraveled with this technique $[4,5]$. Since the overstretching transition bears similarity to thermal melting of DNA, it can be used to study the kinetics and thermodynamics of protein-DNA interaction $[6,7]$.

Yet the mechanical control over a single molecule can also be exploited to study the functioning of biomolecules more in a biological context. For instance, DNA polymerase, the enzyme responsible for the duplication of the genome during cell division, can be forced to reverse its polymerization direction by controlling the tension in the DNA along which it moves [8]. This tells researchers a lot about the how enzymes convert chemical energy ('fuel') into mechanical energy ('movement'). Similarly, DNA tension was recently used to reveal how enzymes, required for the repair of damaged DNA, function [9]. Obviously, optical-tweezers-based force spectroscopy is not limited to the study of DNA and related enzymes; the list of proteins successfully studied using optical tweezers would be too long to be included in this report.

Notably, the NanoTracker ${ }^{\text {mw }}$ is the first commercial optical tweezers platform that offers a complete solution for such quantitative force spectroscopy measurements. To this end, it integrates versatile manipulation, (fluorescence) visualization, and sample control through microfluidics. JPK's vision is to have optical-tweezers technology transcend the labs of self-building enthusiasts and become available for a wider group of scientists.

\section{References}

[1] S. B. Smith, L. Finzi, and C. Bustamante. Direct mechanical measurements of the elasticity of single DNA molecules by using magnetic beads. Science, 258(5085):1122-1126, 1992.

[2] S. B. Smith, Y. Cui, and C. Bustamante. Overstretching B-DNA: the elastic response of individual double-stranded and single-stranded DNA

tural Domains. Journal of Molecular Biology, 327(3):571-578, 2003.

[7] I. D. Vladescu, M. J. McCauley, M. E. Nunez, I. Rouzina, and M. C. Williams. Quantifying forcedependent and zero-force DNA intercalation by single-molecule stretching. Nature Methods, 4(6):517-522, 2007.

[8] G. J. L. Wuite, S. B. Smith, M. Young, D. Keller, and C. Bustamante. Single-molecule studies of the effect of template tension on T7 DNA polymerase activity. Nature, 404(6773):103-106, 2000.

[9] J. van Mameren, M. Modesti, R. Kanaar, C. Wyman, G. J. L. Wuite, and E. J. G. Peterman. Counting RAD51 proteins disassembling from nucleoprotein filaments under tension. Nature, in press, 2008.

Note: This article was first published in the $4^{\text {th }}$ issue of JPK's regular electronic newsletter.

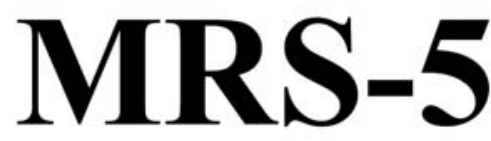

\section{We are ISO-9000 certified and ISO-17025 accredited Microscopy Calibration Standard Now you can calibrate from $1,000 X$ to $1,000,000 X$ !}

This is our fourth generation, traceable, magnification reference standard for all types (SEM, FESEM, Optical, STM, AFM, etc.) of microscopy. The MRS-5 has multiple $\mathrm{X}$ and $\mathrm{Y}$ pitch patterns ranging from $80 \mathrm{~nm}( \pm 1 \mathrm{~nm})$ to $2 \mu \mathrm{m}$ and 3 bar targets from $80 \mathrm{~nm}$ to $3 \mu \mathrm{m}$.

There is also a STM test pattern.

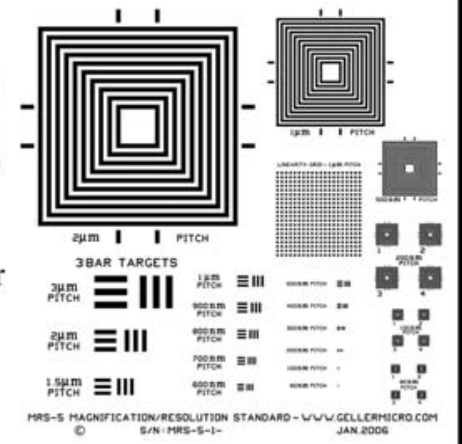

Free web resource guide!

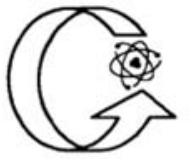

\section{GELLER MICROÅNALYTICAL LABORATORY, Inc.}

426e Boston St., Topsfield, Ma 01983 www.gellermicro.com 